\title{
Predicting Carriage of Extended-Spectrum Beta-Lactamase producing Enterobacteriacae (ESBL-E) in Intensive Care Unit Patients: A Cross Sectional Study
}

\author{
G. Sucila Thangam ${ }^{1 *}$, G. Velvizhi ${ }^{2}$ and C. Revathy ${ }^{2}$ \\ ${ }^{1}$ Department of Microbiology, Madurai Medical College, \\ Madurai - 625020, Tamil Nadu, India \\ ${ }^{2}$ Department of Microbiology, Tirunelveli Medical College, \\ Tirunelveli - 627 011, Tamil Nadu, India \\ *Corresponding author
}

\section{A B S T R A C T}

\begin{tabular}{l} 
Ke y w o r d s \\
$\begin{array}{l}\text { Extended-Spectrum } \\
\text { Beta-Lactamase } \\
\text { Producing } \\
\text { Enterobacteriacae } \\
\text { (ESBL-E) }\end{array}$ \\
\hline Article Info \\
$\begin{array}{l}\text { Accepted: } \\
10 \text { November } 2019 \\
\text { Available Online: } \\
10 \text { December } 2019\end{array}$ \\
\hline
\end{tabular}

The incidence of Extended-Spectrum Beta-Lactamase Producing Enterobacteriacae (ESBL-E) infection is rising worldwide. ESBL-E is a group of pathogens increasingly encountered in ICU setting. Infections with ESBL-E are associated with increased morbidity and mortality. The present study was undertaken to determine the prevalence of patients colonized with Extended Spectrum Beta Lactamase producing Enterobacteriaceae (ESBL-E) in intensive care unit (ICU) patients and also to assess risk factors for ESBL carriage. Patients admitted or transferred to ICU were included in the study and their rectal swabs were collected on admission and discharge. The swabs were cultured and tested for ESBL-E. Out of 50 cases, $41(82 \%)$ cases were Males and 9 (18\%) cases were Females. Most of cases was in the age group of 21-30 years and above followed by that of 11-20 years. Out of these 50 cases, 29 (58\%) cases were culture positive (colonised patients) and $21(42 \%)$ cases were Culture negative (non colonised patients). Of the 29 culture-positive cases, Escherichia coli was isolated from 16 (32\%) samples; Klebsiella pneumoniae was isolated from $5(10 \%)$ samples and both were isolated from $8(16 \%)$. The rate of subsequent development of infection was higher in colonized patients (10\%) compared to non colonized patients (2\%). The study revealed high rates of carriage of ESBL producers in patients admitted to the ICU. Prior colonization with ESBL-E could result in their influx and spread in ICU, challenging infection control measures. Screening of the ESBL producers in the ICU's and proper implementation of infection control measures can control the infections in ICU.

\section{Introduction}

$\begin{array}{lrr}\text { Extended } & \text { Spectrum } & \text { Beta } \\ \text { Lactamase producing } & \text { Enterobacteriaceae }\end{array}$

$\begin{array}{lrr}\text { Extended } & \text { Spectrum } & \text { Beta } \\ \text { Lactamase producing } & \text { Enterobacteriaceae }\end{array}$
(ESBL-E) are found worldwide with rates varying between countries and institutions (Pena et al., 2006; Canton and Coque, 2006). In intensive care unit (ICU), infection and 
colonization by resistant Gram-negative bacteria increase costs, length of stay and mortality. Extended Spectrum Beta Lactamase producing Enterobacteriaceae (ESBL-E) is a group of pathogens increasingly encountered in ICU setting. Conditions that promote ESBL-E acquisition are not completely understood. Infections with ESBL-E are associated with increased morbidity and mortality (Stewardson et al., 2013).

Early detection of ESBL-E carriage could allow timely implementation of infection control measures and the appropriate selection of antimicrobials (Harris et al., 2007). On admission surveillance for ESBL-E has been associated with a reduced incidence of ESBL$\mathrm{E}$ infections during hospitalization (Lowe et al., 2013). However, universal screening for ESBL-E is time consuming, expensive and unlikely to be feasible (Gardam et al., 2002; Thouverez, 2004). Targeted screening based on clinical prediction tools could therefore be useful. The development of such tools requires knowledge of the local epidemiology of ESBL-E (Johnson et al., 2013).

Hence the present study was undertaken to determine the prevalence of patients colonized with Extended Spectrum Beta Lactamase producing Enterobacteriaceae (ESBL-E) in intensive care unit (ICU) patients and also to assess risk factors for ESBL carriage. The main aim of the study is to formulate a prediction tool for ESBL carriage at hospital admission and also to quantify incidence of infections for ICU patients.

\section{Materials and Methods}

This cross sectional study was conducted Department of Microbiology and Department of Medicine (Intensive care unit) at Tirunelveli Medical College Hospital, Tirunelveli, Tamil Nadu for the period of six months from April 2018 to October 2018.The study protocol was approved by the Institutional Scientific and Ethics Committee

\section{Study population}

All patients $>18$ years of age consecutively admitted to Intensive Medical care unit.

\section{Sample collection}

Every patient was underwent rectal swab screening for ESBL-E carriage at admission and then twice a week until ICU discharge performed by ward nurses. A swab is defined as "on admission" if it had been performed within $48 \mathrm{~h}$ of admission to an internal medicine unit. A "discharge swab" was performed within $36 \mathrm{~h}$ of discharge. "Inhospital acquisition" was established if patients had an ESBL-E identified on a discharge swab where the admission swab had been negative. Enhanced hygiene measures (protective gowns, gloves, ESBL-E announcing stickers) were applied in the case of patients colonized and/or infected by ESBL-E and preventively in patients considered at risk for ESBL-E carriage. Demographic data was collected. Patient records were reviewed retrospectively to obtain data on co-morbid conditions and antibiotic use.

\section{Microbiological Methods}

\section{Screening test for ESBLs}

Screening for ESBL-E was performed by inoculating rectal swabs directly onto HiChrome ESBL agar and was incubated at $37^{\circ} \mathrm{C}$ for $18-24 \mathrm{hrs}$. Antibiotic susceptibility was tested using Disc diffusion method on Mueller-Hinton agar according to CLSI guidelines. The following antibiotics were tested: Ampicillin, Cefoxitin, Cefotaxime, Ceftazidime, Imipenem, Ertapenem, 
Meropenem, Gentamicin, Amikacin, Nalidixic acid, Ciprofloxacin and Co-trimoxazole.

\section{Confirmatory tests for ESBLs}

The ESBL producers were confirmed by simultaneously performing the Clinical and Laboratory Standards Institute confirmatory test for the detection of ESBL using doubledisk synergy method (CLSI, 2017). This test requires the use of a third-generation cephalosporin antibiotic disk alone and in combination with clavulanic acid.

In this study, a disk of Ceftazidime $(30 \mu \mathrm{g})$ alone and a disk of Ceftazidime + Clavulanic acid $(30 \mu \mathrm{g} / 10 \mu \mathrm{g})$ were used.

Both the disks were placed at least $25 \mathrm{~mm}$ apart, centre to centre, on a lawn culture of the test isolate on Mueller Hinton Agar (MHA) plate and incubated overnight at $37^{\circ} \mathrm{C}$. Difference in zone diameters with and without clavulanic acid was measured. Interpretation: When there is an increase of $\geq 5 \mathrm{~mm}$ in inhibition zone diameter around combination disk of Ceftazidime + Clavulanic acid versus the inhibition zone diameter around Ceftazidime disk alone, it confirms ESBL production. All ESBL-E producing isolates were stored at $-80^{\circ} \mathrm{C}$.

\section{Statistical analysis}

Data from this study was analyzed descriptively using chi squared tests for categorical variables and student $\mathrm{t}$ tests for interval variables.

Factors potentially associated with previously unknown carriage of ESBL-E on admission and ESBL-E acquisition on discharge was first evaluated using univariate logistic regression. Variables were retained if the P-value was $<0.2$.

\section{Results and Discussion}

Fifty patients were included in the study and their rectal swabs were cultured. Out of these 50 cases, $41(82 \%)$ cases were Males and 9 $(18 \%)$ cases were Females. Most of cases was in the age group of 21-30 years and above followed by that of 11-20 years. Age wise Distribution of Samples was depicted in Table 1.

Out of these 50 cases, 29 (58\%) cases were culture positive (colonised patients) and 21 (42\%) cases were Culture negative (non colonised patients). Of the 29 culture-positive cases, Escherichia coli was isolated from 16 (32\%) samples; Klebsiella pneumoniae was isolated from $5(10 \%)$ samples and both were isolated from $8(16 \%)$ by HiChrome ESBL agar method (Table 2).

The overall prevalence rate of ESBL-E was $58 \%$.The most commonly isolated organism was E. coli (32\%) isolates, Klebsiella isolates $(10 \%)$ and both (16\%) were ESBL-E positive by Double disc diffusion method (Table 3).

All 29 ESBL isolates were resistant to 3rd Generation cephalosporins, Trimethoprimsulfamethoxazole, Ciprofloxacin while resistance rates to Amikacin, Gentamicin, Ampicillin were $86.2 \%$ (25/29), 93.1\% (27/29), 86.2\% (25/29) respectively (Table 4). All isolates were susceptible to Imipenem, Meropenem and Ertapenem.

All the fifty patients screened were followed for the development of infection during ICU period and $4(8 \%)$ developed Blood stream infection in colonized patients. No infection developed in non colonized patients.

The widespread indiscriminate use of antimicrobials has led to the emergence and spread of antibiotic resistance. The prevalence of ESBL-producing isolates has increased in 
the past few decades. Outbreaks of infections in the health-care settings have been linked to higher rates of faecal carriage of ESBLproducing isolates.

The present study showed a rate of $58 \%$ faecal carriage of ESBL producers. The prevalence of faecal carriage of ESBLs in this study was similar to the study conducted by Mulki et al., (2017). In a study done by Paniagua et al., (2010) the rates of faecal carriage of ESBL producers had increased from $0.3 \%$ to $11.8 \%$ from 1991 to 2003. The higher rate of carriage in this study may be due to increased exposure of antibiotics over the years. All the fifty patients screened were followed for the development of infection during ICU period and $4(8 \%)$ developed Blood stream infection in colonized patients. No infection developed in non colonized patients. The previous studies have reported $65 \%$ carriage rate in patients with urinary tract infection and $8.5 \%$ carriage rate in healthy individuals (Rodríguez-Baño et al., 2008). A study by Reddy et al., showed that $8.5 \%$ of the ESBL colonizers developed a subsequent ESBL bloodstream infections (Reddy, 2007).

Table.1 Age wise distribution of samples

\begin{tabular}{|c|c|}
\hline Age & No of patients \\
\hline $\mathbf{1 1 - 2 0}$ & 11 \\
\hline $\mathbf{2 1 - 3 0}$ & 13 \\
\hline $\mathbf{3 1 - 4 0}$ & 11 \\
\hline $\mathbf{4 1 - 5 0}$ & 6 \\
\hline $\mathbf{5 1 - 6 0}$ & 4 \\
\hline $\mathbf{6 1 - 7 0}$ & 3 \\
\hline$<\mathbf{7 0}$ & 2 \\
\hline
\end{tabular}

Table.2 Organism wise distribution of samples

\begin{tabular}{|c|c|c|}
\hline Organism & No of growth & Percentage \\
\hline Escherichia coli & 16 & $32 \%$ \\
\hline $\begin{array}{c}\text { Klebsiella pneumoniae } \\
\text { Escherichia coli\& Klebsiella } \\
\text { pneumoniae }\end{array}$ & 5 & $10 \%$ \\
\hline
\end{tabular}

Table.3 Frequency of ESBL production in Gram negative isolates among carriers

\begin{tabular}{|c|c|c|}
\hline Sl. no & Organism & Percentage \\
\hline $\mathbf{1}$ & Escherichia coli & $32 \%$ \\
\hline $\mathbf{2}$ & Klebsiella pneumoniae & $10 \%$ \\
\hline $\mathbf{3}$ & Escherichia coli\& Klebsiella pneumoniae & $16 \%$ \\
\hline
\end{tabular}


Table.4 Proportion of anti microbial resistance among 29 ESBL producing Escherichia coli and Klebsiella pneumoniae

\begin{tabular}{|c|c|c|}
\hline Anti microbial agent & E.coli & $\begin{array}{c}\text { Klebsiella } \\
\text { pneumoniae }\end{array}$ \\
\hline Amikacin & $81.25 \%$ & $92.3 \%$ \\
\hline Gentamicin & $93.75 \%$ & $92.3 \%$ \\
\hline Ciprofloxacin & $100 \%$ & $100 \%$ \\
\hline $\begin{array}{c}\text { Trimethoprim- } \\
\text { sulfamethoxazole }\end{array}$ & $100 \%$ & $100 \%$ \\
\hline Ampicillin & $100 \%$ & $69.2 \%$ \\
\hline
\end{tabular}

The rates of colonization with multi resistant isolates have shown an alarming rise over the past few decades. The matter of concern is the emergence and spread of ESBL-producing isolates. The rectal surveillance for ESBLproducing Enterobacteriaceae will play a pivotal role in infection control. The ESBLcolonized patients act as source of infection to the other hospitalized patients in high-risk areas.

Asymptomatic colonization of ESBLs can thus be a threat to the subsequent development of infection, especially in the ICU settings. The surveillance of rates of faecal carriage of ESBL-producing Enterobacteriaceae may be helpful to track and monitor the spread of these organisms in the hospital settings. The rates of infection can be controlled by following strict infection control measures.

This study focuses on screening for faecal carriage of ESBL-producing Enterobacteriaceae members using rectal swab cultures in patients admitted to ICU of a tertiary care hospital and follows the patients for the subsequent development of infections with ESBL-producing organisms in the gut flora. The study revealed high rates of carriage of ESBL producers in patients admitted to the ICU. Prior colonization with ESBL-E could result in their influx and spread in ICU, challenging infection control measures. Gut colonization with these strains may be a source of endogenous infection and horizontal transfer of these genes in future. Carriage of ESBL-producing commensal strains of Enterobacteriaceae is a threat as it can get transferred to the pathogenic strains. Screening of the ESBL producers in the ICU's and proper implementation of infection control measures can control the infections in ICU.

\section{Acknowledgement}

The authors are gratefully acknowledge The Dean, Tirunelveli Medical College Hospital, Tirunelveli, Tamil Nadu and The Staff of Microbiology and Medicine Department of Tirunelveli Medical College Hospital.

\section{References}

Canton, R, Coque, T.M.(2006) The CTX-M beta-lactamase pandemic. Curr Opin. Microbiol 9: 466-475.

Clinical Laboratory Standards Institute; Performance standards for antimicrobial susceptibility testing. $28^{\text {th }}$ Informational Supplement: 2017.

Gardam, M.A., Burrows, L.L., Kus, J.V., Brunton, J., Low, D.E., Conly, J.M,et al.,(2002) Is surveillance for multidrug-resistant Enterobacteriaceae an effective infection control strategy in the absence of an outbreak? J Infect Dis 186: 1754-1760. 
Harris, A.D., Kotetishvili, M., Shurland, S., Johnson, J.A., Morris, J.G., Nemoy, L. L., et al., (2007). How important is patient-to-patient transmission in extended-spectrum beta-lactamase Escherichia coli acquisition. Am J Infect Control 35: 97-101.

Johnson, S.W., Anderson, D.J., May, D.B., Drew, R.H. (2013). Utility of a clinical risk factor scoring model in predicting infection with extended-spectrum beta lactamase-producing

enterobacteriaceae on hospital admission. Infect Control Hosp Epidemiol 34: 385-392.

Lowe,C.F., Katz, K., McGeer, A.J., Muller, M.P. (2013) Efficacy of admission screening for extended-spectrum Betalactamase producing Enterobacteriaceae. PLoS One 8:e62678.

Mulki, S.S., Kavya, R., Sevitha, B. (2017). Faecal Carriage of Extended-spectrum Beta-lactamase-producing Enterobacteriaceae in Intensive Care Unit Patients. Ind $\mathbf{J}$ Critical Care Medicine. 21(8): 525-527.

Paniagua, R., Valverde, A., Coque, T.M., Baquero, F., Cantón, R. (2010) Assessment of prevalence and changing epidemiology of extendedspectrum beta-lactamase-producing Enterobacteriaceae faecal carriers using a chromogenic medium. Diagn Microbiol Infect Dis. 67: 376-9.

Pena, C., Gudiol, C., Tubau, F., Saballs, M., Pujol, M., Dominguez, M.A., et al., (2006) Risk-factors for acquisition of
Extended-Spectrum Beta lactamaseproducing Escherichia coli among hospitalised patients. Clin Microbiol Infect 12: 279-284.

Reddy, P., Malczynski, M., Obias, A., Reiner, S., Jin N, Huang J, et al.,(2007) Screening for Extended-Spectrum Beta-Lactamase-producing

Enterobacteriaceae among high-risk patients and rates of subsequent bacteraemia. Clin Infect Dis. 45:84652.

Rodríguez-Baño, J., López-Cerero, L., Navarro, M.D., Díaz de Alba, P., Pascual (2008) A. Faecal carriage of Extended-Spectrum Beta-Lactamaseproducing Escherichia coli: Prevalence, risk factors and molecular epidemiology. J Antimicrob Chemother. 62: 1142-9.

Stewardson, A., Fankhauser, C., De Angelis, G., Rohner, P., Safran, E., Schrenzel, J,et al.,(2013) Burden of bloodstream infection caused by Extended Spectrum Beta Lactamase-producing Enterobacteriaceae determined using multistate modelling at a Swiss University Hospital and a nationwide predictive model. Infect Control Hosp Epidemiol 34:133-143.

Thouverez, M., Talon, D., Bertrand,X.(2004) Control of Enterobacteriaceae producing Extended-Spectrum BetaLactamase in intensive care units: Rectal screening may not be needed in non-epidemic situations. Infect Control Hosp Epidemiol 25:838-841.

\section{How to cite this article:}

Sucila Thangam, G., G. Velvizhi and Revathy, C. 2019. Predicting Carriage of ExtendedSpectrum Beta-Lactamase Producing Enterobacteriacae (ESBL-E) in Intensive Care Unit Patients: A Cross Sectional Study. Int.J.Curr.Microbiol.App.Sci. 8(12): 1010-1015. doi: https://doi.org/10.20546/ijcmas.2019.812.129 